\title{
On heat transfer and evaporation characteristics in the growth process of a bubble with microlayer structure during nucleate boiling
}

\author{
Zhihao Chen* and Yoshio Utaka \\ Faculty of Engineering, Yokohama National University, Tokiwadai 79-5, Hodogaya, Yokohama \\ 240-8501, Japan
}

We have previously observed and experimentally measured a microlayer formed beneath a growing bubble during nucleate pool boiling. The initial microlayer thickness was of micrometer order and increased linearly with distance from the bubble inception site. The quantitative degree of contribution of the microlayer evaporation to bubble growth was still not elucidated, although a large number of experimental studies have been conducted on the distribution and evaporation characteristics of the microlayer. To clarify the heat transfer characteristics, especially the contribution of microlayer evaporation in nucleate pool boiling, numerical simulations are performed here for two phase vapor-liquid flow induced by the growth of a single bubble using the volume of fluid method. Furthermore, a special model is proposed to combine the microlayer and bulk liquid regions, the scales of which are extremely different in the simulations. The microlayer was neglected in the volume fraction calculation, while the evaporation from the microlayer was included by applying the source terms of the basic equations for the presence of a virtual microlayer. Similar tendencies were observed between the calculated and experimental results on the variations in the microlayer radius and bubble volume. The proportion of microlayer evaporation to total bubble volume was generally in agreement with the previous results, and the ratio of microlayer evaporation to the change in the total bubble volume was approximately 40 percent.

Keywords: Numerical simulation, VOF, Nucleate pool boiling, Phase change heat transfer, Microlayer evaporation

\footnotetext{
* Corresponding author. Tel/Fax: +81 453393909.

E-mail: utaka@ynu.ac.jp
} 


\section{Introduction}

Several mechanisms for nucleate boiling have been previously proposed that can be classified into two categories; convective heat transfer and latent heat transport. The latent heat transport mechanism involves the microlayer evaporation mechanism; a thin liquid film (microlayer) exists between a growing bubble and the heat transfer surface, from which a large amount of heat is transported by vaporization of the microlayer. The existence and distribution of the microlayer was confirmed by utilizing several different methods in recent experimental studies, such as laser interferometry [1,2], laser extinction [3-5], and indirect inference of the microlayer thickness based on the measured temperature variation in the heat transfer surface [6]. The initial microlayer thickness was found to increase linearly with the distance from the bubble inception site. Furthermore, Utaka et al. [7] conducted a two-dimensional (2D) numerical calculation on the transient heat conduction of a heat transfer plate with the heat flux of microlayer evaporation as the boundary condition on the backside of the heat transfer surface, which is calculated based on the surface superheat at bubble inception $\Delta T_{\mathrm{i}}$, and the distribution of initial microlayer thickness $\delta_{0}$, measured from experiments with water and ethanol. Evaporation of the microlayer and the consequent decrease in its thickness were discussed; however, instead of calculating the evaporation from the superheated liquid layer, the bubble volume acquired from a recorded image of the bubble was adopted to determine the contribution of microlayer evaporation to the total amount of evaporation during the bubble growth process. As a result, the proportion of evaporation from the microlayer to the bubble volume was approximately 20-70\%, as shown in Fig. 1, and this increased with the surface superheat at bubble inception, $\Delta T_{\mathrm{i}}$. It was demonstrated that both evaporation from the microlayer and the superheated liquid layer are closely related to the bubble growth in saturated nucleate boiling. Therefore, it is necessary to quantitatively evaluate both the evaporation from the microlayer and that from the superheated liquid layer to elucidate the mechanism of nucleate boiling.

In the growth process of a boiling bubble, the temperature distribution in the vicinity of the vapor-liquid interface changes rapidly due to evaporation and the extremely fast movement of the interface with growth of the bubble. In this study, the heat transfer characteristics of the bubble growth process during nucleate boiling are investigated numerically. The volume of fluid (VOF) method developed by Hirt and Nichols [8] is an effective approach for interface capturing of free surface flow and its modified versions have been widely adopted in research on two-phase flow (e.g. boiling). Stephan and coworkers [9,10] established a new steady state model for calculating the evaporation from a liquid microfilm and performed numerical simulations on nucleate pool boiling. The microfilm referred to here is a thin liquid film between the bulk liquid and absorbed film on the heat transfer surface. The absorbed film is extremely thin (a few molecular layers thick). The evaporation from this microfilm region was calculated and the heat transfer characteristics during the growth of a single bubble were investigated. A large amount of evaporation was determined to occur in the microfilm region during the boiling process. However, the microfilm region in the simulations is extremely narrow and was different from the 
experimentally measured microlayer region examined in the study. Therefore, the experimentally elucidated microlayer was not included in the numerical simulation. Ose et al. [11] conducted a numerical simulation on the bubble behavior in subcooled boiling by utilizing an improved phase-change model based on the temperature-recovery method [12]. It was concluded that a model with consideration of the relaxation time based on unsteady heat conduction could predict the bubble growth and condensation processes in the subcooled pool boiling phenomena. Jiang et al. [13] performed a numerical simulation on the bubble growth and heat transfer in nucleate boiling using a hybrid scheme [14] that combined the mechanical boiling model with a computational fluid dynamics (CFD) simulation. However, a similar structure to that from the simulation of Stephan et al. [9] was adopted for the liquid film between the bubble and heat transfer surface. The calculation results for bubble growth (bubble radius) were then approximated in accordance with the experimental results. For the various research summarized here, although the calculation results were compared with the experimental results, the experimentally measured microlayer was not considered in the numerical simulations. Therefore, it is necessary to acquire qualitative knowledge regarding microlayer evaporation and its contribution to bubble growth to elucidate the mechanisms and heat transfer characteristics of nucleate pool boiling.

In this study, the microlayer present between a growing bubble and the heat transfer surface is extremely thin compared with the bubble size; therefore, it is difficult to simulate the microlayer region. To solve this problem, a special model is proposed, in which the microlayer is ignored in the main calculation of the macroscopic bubble and a hypothetical microlayer is applied to the calculation. The evaporation from the microlayer and the superheated liquid layer was computed separately. A 2D numerical simulation is conducted in an axisymmetric domain on the two phase vapor-liquid flow induced by the growth of a single bubble during nucleate boiling using the VOF method. Evaporation from the microlayer and superheated liquid layer, and the bubble behavior and heat transfer characteristics during the bubble growth process are closely analyzed. In particular, to consider the influence of microlayer formation beneath the bubble, the experimentally measured initial distribution of the microlayer and the surface superheat at bubble inception $\Delta T_{\mathrm{i}}$ [5] are applied to the simulations. Evaporation of the microlayer is calculated based on a 1D quasi-steady heat conduction of the microlayer. Moreover, the evaporation from the superheated liquid layer is calculated according to the temperature gradient at the vapor-liquid interface, and is applied to the numerical simulation together with that from the microlayer.

\section{Nomenclature}

$\begin{array}{ll}A & \text { area }\left(\mathrm{m}^{2}\right) \\ C & \text { specific heat }(\mathrm{J} / \mathrm{kg} \cdot \mathrm{K}) \\ C_{r} & \text { Courant number } \\ F & \text { volume fraction of fluid }\end{array}$


$f_{r}$

$f_{z}$

$g$

$h_{i}$

L

$\Delta L$

p

$p_{s}$

$\dot{m}$

$q$

$q_{s}$

$r$

$R_{\mathrm{M}}$

$R_{\mathrm{B}}$

$R_{\text {Mmax }}$

$s_{f}$

$s_{m}$

T

$\Delta T_{\mathrm{i}}$

$t$

$t_{\mathrm{B}}$

$t_{\mathrm{Bd}}$

$\Delta t$

$V_{\mathrm{ML}}$

$V_{\text {B }}$

$v_{r}$

$v_{z}$

Z

\section{Greek}

$\theta$

$\theta_{\text {a }}$

$\theta_{\mathrm{c}}$

$\delta$

$\rho$

$\lambda$ source term of Navier-Stokes equation in radial direction

source term of Navier-Stokes equation in axial direction

acceleration of gravity $\left(\mathrm{m} / \mathrm{s}^{2}\right)$

evaporation heat transfer coefficient $\left(\mathrm{W} / \mathrm{m}^{2} \cdot \mathrm{K}\right)$

latent heat of vaporization $(\mathrm{J} / \mathrm{kg})$

normal distance from superheated liquid cell to interface (m)

pressure $(\mathrm{Pa})$

set value of pressure $(\mathrm{Pa})$

mass flux $(\mathrm{kg} / \mathrm{s})$

heat flux $\left(\mathrm{W} / \mathrm{m}^{2}\right)$

source term of energy equation

radial direction (distance from bubble inception site in radical direction; $\mathrm{mm}$ )

microlayer radius on heat transfer surface $(\mathrm{mm})$

bubble radius $(\mathrm{mm})$

maximum microlayer radius ( $\mathrm{mm}$ )

source term of volume fraction equation

source term of continuity equation

temperature $(\mathrm{K})$

surface superheat temperature at bubble inception (K)

time (s)

elapsed time from bubble inception (ms)

elapsed time from bubble inception to completion of bubble departure (ms)

time step (s)

evaporation from microlayer $\left(\mathrm{mm}^{3}\right)$

bubble volume $\left(\mathrm{mm}^{3}\right)$

radial velocity $(\mathrm{m} / \mathrm{s})$

axial velocity $(\mathrm{m} / \mathrm{s})$

axial direction (distance from bubble inception site in axial direction; mm)

non-dimensional temperature (-)

apparent contact angle $\left({ }^{\circ}\right)$

angle of reference position on the vapor-liquid interface $\left(^{\circ}\right)$

microlayer thickness $(\mu \mathrm{m})$

density $\left(\mathrm{kg} / \mathrm{m}^{3}\right)$

thermal conductivity $(\mathrm{W} / \mathrm{m} \cdot \mathrm{K})$ 


$$
\begin{array}{cr}
\mu & \text { viscosity (Pa·s) } \\
\sigma & \text { evaporation coefficient (-) } \\
\eta & \text { non-dimensional distance in axial direction (-) }
\end{array}
$$

$\begin{array}{ll}\text { Subscript } & \\ \text { BK } & \text { bulk liquid } \\ \text { cell } & \text { calculation cell } \\ \text { ex } & \text { experimental value } \\ \text { ML } & \text { microlayer } \\ \text { L } & \text { liquid } \\ \text { V } & \text { vapor } \\ \text { sat } & \text { saturation state } \\ \text { surface } & \text { vapor-liquid interface } \\ \text { w } & \text { heat transfer surface }\end{array}$

\section{Superscript}

$0 \quad$ initial

\section{Numerical simulation model}

\subsection{Calculation domain}

In this study, a numerical simulation was conducted for the heat transfer characteristics and behavior of a single bubble during saturated nucleate boiling of water at atmospheric pressure. The calculation domain is a 2D axisymmetric domain including fluid and solid regions that has a radius of $6 \mathrm{~mm}, 10 \mathrm{~mm}$ height for the fluid region and $2 \mathrm{~mm}$ for the solid region, as shown in Fig. 2. Quartz glass is set as the solid region material, in accordance with the experimental apparatus, while water (vapor and liquid) is set as the e fluid region material. The physical properties of vapor and liquid at the saturation state under atmospheric pressure were adopted in the numerical calculations.

\subsection{Governing equations and boundary conditions}

The following governing equations were employed in the numerical simulations. The fluid is treated as incompressible.

\subsubsection{Governing equations}

Equation of continuity:

$$
\frac{\partial \rho}{\partial t}+\frac{1}{r} \frac{\partial}{\partial r}\left(r \rho v_{r}\right)+\frac{\partial}{\partial z}\left(\rho v_{z}\right)=s_{m} .
$$


Navier-Stokes equation:

$\rho\left(\frac{\partial v_{r}}{\partial t}+v_{r} \frac{\partial v_{r}}{\partial r}+v_{z} \frac{\partial v_{r}}{\partial z}\right)=-\frac{\partial p}{\partial r}+\mu\left(\frac{\partial^{2} v_{r}}{\partial z^{2}}+\frac{1}{r} \frac{\partial}{\partial r}\left(r \frac{\partial v_{r}}{\partial r}\right)-\frac{v_{r}}{r^{2}}\right)+f_{r}$

$\rho\left(\frac{\partial v_{z}}{\partial t}+v_{r} \frac{\partial v_{z}}{\partial r}+v_{z} \frac{\partial v_{z}}{\partial z}\right)=-\frac{\partial p}{\partial z}+\mu\left(\frac{\partial^{2} v_{z}}{\partial z^{2}}+\frac{1}{r} \frac{\partial}{\partial r}\left(r \frac{\partial v_{z}}{\partial r}\right)\right)+\rho g+f_{z}$

Energy equation:

$\rho c\left(\frac{\partial T}{\partial t}+v_{r} \frac{\partial T}{\partial r}+v_{z} \frac{\partial T}{\partial z}\right)=\left[\frac{1}{r} \frac{\partial}{\partial r}\left(r \lambda \frac{\partial T}{\partial r}\right)+\frac{\partial}{\partial z}\left(\lambda \frac{\partial T}{\partial z}\right)\right]+q_{s}$.

Volume fraction equation:

$$
\begin{aligned}
& \frac{\partial F_{\mathrm{L}}}{\partial t}+v_{r} \frac{\partial F_{\mathrm{L}}}{\partial r}+v_{z} \frac{\partial F_{\mathrm{L}}}{\partial z}=s_{f}, \\
& F_{\mathrm{L}}+F_{\mathrm{V}}=1 .
\end{aligned}
$$

The VOF method was adopted for the simulations using the commercial software CFD solver ANSYS Fluent version 13 [15]. The convective term in the volume fraction equation was computed through a geometrical piecewise linear interface calculation (PLIC) [16] reconstruction algorithm, in which the direction of the vapor-liquid interface was taken into account. The surface tension at the interface was treated as a body force using the continuum surface force (CSF) method proposed by Brackbill et al. [17]. The convective terms in the Navier-Stokes and energy equations were discretized with a second-order upwind difference scheme, while the diffusive terms were discretized with a second-order central finite-difference scheme. A body-force-weighted scheme [15] was adopted to interpolate cell-centered pressures at the cell faces. The cell-centered gradients of each scalar field were computed using a least squares cell based scheme [15]. The pressure-velocity coupling was handled with a pressure implicit splitting of operators (PISO) algorithm [18], which converges more quickly than the other options available in the solver. A first-order explicit scheme was adopted for the time discretization of the volume fraction equation, while a first-order implicit scheme was adopted for other basic equations. Moreover, for the transient problem calculated using VOF method, various time steps were adopted for calculation of the volume fraction equation and other transport equations. The time step is determined according to the maximum Courant number $\left(C_{r}\right)$ allowed for the interface and near-interface cells. The Courant number compares the time step $\Delta t$, and the time taken for the fluid to empty out of the cell (Eq. 7). In this study, the maximum Courant number was set to 0.05 for calculation of the volume fraction equation and 0.1 for other transport equations.

$$
C_{r}=\frac{\Delta t}{V_{\text {cell }} / \sum v_{\text {fluid }}}
$$




\subsubsection{Boundary conditions}

Boundary conditions were applied based on Eqs. 8-11. The experimental heat flux $q_{\mathrm{ex}}$, was applied at the bottom of the calculation domain. The pressure outlet boundary condition [15] was applied to the boundary of the fluid region to simulate outflow and backflow of the liquid induced by growth and detachment of the bubble. The backflow temperature was set as the average temperature distribution of fluid, which is introduced in chapter 2.5. In addition, a half-spherical bubble with a radius of $0.15 \mathrm{~mm}$ was arranged as the initial bubble on the center position of heat transfer surface in the fluid region.

$$
\begin{array}{ll}
r=6 \mathrm{~mm}, \mathrm{z}=-2 \sim 0 \mathrm{~mm}: & q=0 \\
\mathrm{z}=-2 \mathrm{~mm}: & q=q_{\mathrm{ex}} \\
r=6 \mathrm{~mm}, \mathrm{z}=0 \sim 10 \mathrm{~mm}: & \frac{\partial v_{r}}{\partial r}=0, \frac{\partial v_{z}}{\partial r}=0, \frac{\partial T}{\partial r}=0 \\
\mathrm{z}=10 \mathrm{~mm}: & \frac{\partial v_{r}}{\partial z}=0, \frac{\partial v_{z}}{\partial z}=0, \frac{\partial T}{\partial z}=0
\end{array}
$$

\subsection{Calculation model for evaporation at the vapor-liquid interface}

\subsubsection{Microlayer evaporation}

The microlayer that is between a growing bubble and the heat transfer surface is extremely thin compared with the bubble size. Therefore, it is difficult to simulate the microlayer region in the calculation for the macroscopic bubble using the VOF method, so that special handling is necessary for simulation of the microlayer. In this study, a special model is proposed where the evaporation from the microlayer and superheated liquid layer are computed separately. The microlayer was ignored in the volume fraction of fluid calculation, while vapor generation from the microlayer and the corresponding variation in momentum and heat were computed and applied to the source terms of the respective governing equations. Specifically, a hypothetical microlayer, as shown in Fig. 3, was applied and arranged to the calculation cells adjacent to the heat transfer surface that are full of vapor $\left(F_{\mathrm{V}}=1\right)$, and the experimentally measured distribution of initial microlayer thickness (Eq. 12) [5] was adopted. The heat flux of microlayer evaporation (Eq. 13) was determined based on the overall coefficient of heat transfer, which could be calculated based on $1 \mathrm{D}$ heat conduction of the microlayer and the interfacial evaporation heat transfer coefficient, $h_{i}$ (Eq. 14). The evaporation coefficient $\sigma$, was assigned as 1 for the calculation of $h_{\mathrm{i}}$. The mass flow rate of evaporation (Eq. 15) and the decrease in microlayer thickness (Eq. 16) were then calculated based on the obtained heat flux.

$$
\begin{aligned}
& \delta_{\mathrm{ML}}^{0}=4.46 \times 10^{-3} \times r \\
& q_{\mathrm{ML}}=\frac{\left(T_{\mathrm{w}}-T_{\mathrm{sat}}\right)}{\delta / \lambda_{\mathrm{L}}+1 / h_{i}}
\end{aligned}
$$




$$
\begin{aligned}
& h_{i}=\frac{2 \sigma}{2-\sigma} \frac{L^{2} \rho_{v}}{\sqrt{2 \pi R T_{\text {sat }}^{3}}} \\
& \dot{m}_{\mathrm{ML}}=\frac{q_{\mathrm{ML}} A_{\text {surface }}}{L} \\
& \Delta \delta_{\mathrm{ML}}=-\frac{q_{\mathrm{ML}} \Delta t}{\rho_{\mathrm{L}} L}
\end{aligned}
$$

Moreover, when the decrease in microlayer thickness in one time step exceeded the thickness of the microlayer in the calculation cell, the heat flux and the corresponding mass flow rate of microlayer evaporation were adjusted to zero the microlayer thickness. The computed mass flow rate was then applied to the source terms of the continuity equation and volume fraction equation as the vapor generation from the microlayer. Furthermore, the momentum and heat generation corresponding to the mass flow rate were also computed and applied to the source terms of the Navier-Stokes equation and energy equation, respectively. The evaporative latent heat corresponding to the amount of evaporation was applied to the source term (negative) of the energy equation in the calculation cells of the heat transfer surface adjacent to the microlayer.

\subsubsection{Evaporation from surrounding bulk liquid}

Besides the microlayer region, vigorous evaporation also occurs in the bulk superheated liquid layer near the heat transfer surface. The evaporation from surrounding bulk liquid should also be calculated in the simulation. In this study, a method similar to that for determining the microlayer evaporation was adopted to calculate the heat flux for evaporation from the bulk superheated liquid layer, which was determined based on the overall heat transfer coefficient (Eq. 17).

$$
q_{\mathrm{BK}}=\frac{T_{\mathrm{L}}-T_{\mathrm{sat}}}{\Delta L / \lambda_{\mathrm{L}}+1 / h_{i}}
$$

Firstly, for a certain cell of vapor-liquid interface, tThe exact position and direction of the interface is necessary to be decided for computing the heat flux using Eq. 17; A PLIC (piecewise linear interface calculation) based algorithm has been proposed to capture the exact position of the vapor-liquid interface [19]. Then, it is necessary to select a liquid cell $\left(F_{\mathrm{L}}=1\right)$ from the surrounding cells for determining the degree of superheat of the surrounding liquid $\left(\Delta T_{\mathrm{sat}}=T_{\mathrm{L}}-T_{\mathrm{sat}}\right)$, and the normal distance from the superheated liquid cell to the interface, while the evaporation heat transfer coefficient $h_{i}$, could be calculated using Eq. 14. In order to determine the superheated liquid cell from the eight surrounding cells for the heat flux calculation, two typical cases are divided according to the inclination of the vapor-liquid interface, as shown in Fig. 4(a) and (b). Case 1: for the interface with relatively large gradient (the condition was set as the angle between the vapor-liquid interface and the axis is in the range of $15-75^{\circ}$ ) as 
shown in Fig. 4(a), the liquid cell $\left(F_{\mathrm{L}}=1\right)$ near the normal line passing through the center of the vapor-liquid interface is adopted. Case 2: for the interface with relatively small gradient (the angle between the vapor-liquid interface and the axis is out of the range of $15-75^{\circ}$ ) as shown in Fig. 4(b), the liquid cell $\left(F_{\mathrm{L}}=1\right)$ adjacent to the interface cell in the normal direction of the vapor-liquid interface was adopted. Besides, it is also possible that there is no liquid cell $\left(F_{\mathrm{L}}=1\right)$ presents in the normal direction of vapor-liquid interface in case 2 . In such a case, it was treated as case 1ven though the gradient of the interface is relatively small. After the calculation cell of superheated liquid is determined, the degree of superheat ( $\Delta T_{\text {sat }}=T_{\mathrm{L}}-T_{\text {sat }}$ ) and-the normal distance ( $\left.\Delta L\right)$ from the center point of the supheated liquid cell to the vapor-liquid interface could be determined.

The mass flow rate induced by evaporation could be calculated based on the computed heat flux using Eq. 18.

$$
\dot{m}_{\mathrm{BK}}=\frac{q_{\mathrm{BK}} A_{\text {surface }}}{L}
$$

The obtained mass flow rate and the corresponding variation in momentum and heat are applied to the source terms of the continuity equation, the Navier-Stokes equation and the energy equation. Positive source terms corresponding to vapor generation were applied to the vapor cells $\left(F_{V}=1\right)$ in the vicinity of the vapor-liquid interface, and negative source terms corresponding to liquid extinction were applied to the liquid cells $\left(F_{\mathrm{L}}=1\right)$ in the vicinity of the vapor-liquid interface. The negative source term of the energy equation that corresponds to the evaporative latent heat was applied to the liquid cells $\left(F_{\mathrm{L}}=1\right)$ adjacent to vapor-liquid interface cell. Furthermore, source terms corresponding to vapor generation were smeared over a few cells in the vicinity of the vapor-liquid interface to avoid numerical instability in some cells. In addition, adaptive mesh refinement [15] was conducted in the vicinity of the vapor-liquid interface to improve the computational efficiency. The mesh size for bulk liquid was set as $20 \mu \mathrm{m}$; the minimum mesh sizes adopted for adaptive mesh refinement were 10, 5, 2.5 and $1.25 \mu \mathrm{m}$.

\subsection{Apparent contact angle of bubble}

The microlayer was not calculated in the volume fraction equation. Therefore, the three-phase contact line in the numerical simulation becomes the border of the bulk liquid and the virtual microlayer, which is not coincident with the actual three-phase contact line. This is referred as to as the apparent contact angle of the bubble for the liquid side angle between the vapor-liquid interface and the heat transfer surface in the simulation. The contact angle of water could not be applied as the apparent contact angle. Therefore, the apparent contact angle applied to the numerical simulation was measured from the experimental bubble images. Figure 5 shows a schematic diagram of a bubble. The apparent contact angle applied to the numerical simulation could be calculated based on the bubble radius $R_{\mathrm{b}}$, and the microlayer radius $R_{\mathrm{M}}$ $\left(\theta_{\mathrm{a}}=\arcsin \left(R_{\mathrm{M}} / R_{\mathrm{B}}\right)\right)$, under the assumption of spherical shape, both of which could be measured from the bubble images. Moreover, the apparent contact angle varies during the bubble growth process and has a 
linear relationship with time, as shown in Fig. 6. Therefore, the linear function between the apparent contact angle and elapsed time, which is obtained based on the measured values of the apparent contact angle (circles in Fig. 6), was applied to the numerical simulations.

\subsection{Initial conditions for calculations}

In saturated nucleate pool boiling, a bubble is initially formed and then grows in the superheated liquid layer formed adjacent to the heat transfer surface. The bubble growth is related to the temperature distribution of the superheated layer; therefore, it is necessary to adopt the exact temperature distribution of the liquid region at the bubble inception for the simulations. Fujita et al. [20] experimentally measured the temperature distribution of the liquid for saturated nucleate boiling of water and some organic liquids. They reported that the average liquid temperature decreased linearly with increasing distance from the heat transfer surface, and gradually became close to the bulk liquid temperature from the outer region of the superheated liquid layer. In addition, the dimensionless temperature distribution (calculated using Eqs. 19 and 20) became similar over the entire liquid region, regardless of the region of nucleate boiling and the density of nuclei.

$$
\begin{aligned}
& \theta=1-\eta \quad(0 \leq \eta \leq 0.5), \\
& \theta=\frac{0.257}{\left(\eta^{3 / 2}-0.0883\right)^{1 / 2}} \quad(0.5 \leq \eta) .
\end{aligned}
$$

where $\theta=\frac{T-T_{\text {sat }}}{T_{\mathrm{w}}-T_{\text {sat }}}$ and $\eta=\frac{Z}{\delta} \cdot \delta$ is the thickness of the equivalent layer of heat conduction and can be determined from the following equation.

$$
N u=1.93(R / \delta)
$$

where $N u$ denotes the Nusselt number, $N u=\alpha R / \lambda_{\mathrm{L}}$, and $R, \alpha, \lambda_{\mathrm{L}}$ represent the characteristic length of the heat transfer surface (radius), the heat transfer coefficient and the heat conductivity of the liquid, respectively.

In this study, the initial temperature distribution of the liquid region was calculated using these dimensionless equations. Moreover, the linear temperature distribution was applied as the initial temperature of the heat transfer block, which was calculated based on the heat flux and the surface superheat temperature at bubble inception $\Delta T_{\mathrm{i}}$.

\section{Results and discussion}

As introduced in Sec.1, Utaka et al. [7] investigated the contribution of microlayer evaporation to the total amount of evaporation (Fig. 1). The surface superheat temperature at bubble inception $\Delta T_{\mathrm{i}}$, was obtained by iterative calculation of the $1 \mathrm{D}$ transient heat conduction of the heat transfer plate until the variation in microlayer thickness coincided with the experimentally measured value. The values of $\Delta T_{\mathrm{i}}$ 
were scattered widely. For example, for the conditions of heat flux $q=103 \mathrm{~kW} / \mathrm{m}^{2}$ and maximum microlayer radius $R_{\mathrm{Mmax}}=3.0-3.5 \mathrm{~mm}, \Delta T_{\mathrm{i}}$ was distributed widely in the range of $16-28 \mathrm{~K}$. The reason for this is that the temperature of the heat transfer surface is coupled with the temperature of the superheated liquid layer, which has temporal/spatial variation during the bubble growth process. However, it is difficult to experimentally measure the temperature distributions of the heat transfer surface and liquid region. The average temperature distribution of the liquid region was applied here as the initial temperature distribution, as introduced in chapter 2.5 , and the heat transfer characteristics were analyzed for the average state. Therefore, the experimental results with different initial superheat $\Delta T_{\mathrm{i}}$, distributed around the average superheat were adopted for comparison with the calculated results.

\subsection{Variation in microlayer radius and bubble volume}

The influence of different minimum mesh size is summarized in Fig. 7. The variation in the microlayer radius was influenced by the minimum mesh size, where relatively rapid growth of the microlayer radius was achieved when a smaller minimum mesh size was adopted; the maximum microlayer radius became larger as a consequence. The difference in the microlayer radius between the simulation results for different minimum mesh sizes was getting smaller with the decrease of minimum mesh size. For minimum mesh sizes of 1.25 or $2.5 \mu \mathrm{m}$, the difference between the calculation results is quite small. In addition, the computational consumption becomes larger with decreasing minimum mesh size. In particular, a large amount of time was required for the calculation with a minimum mesh size of $1.25 \mu \mathrm{m}$. Consequently, the calculation results for a minimum mesh size of $2.5 \mu \mathrm{m}$ were adopted for analysis in this study, under the consideration of both the influence of minimum mesh size and the computational consumption.

The experimental and numerical results for the variations in microlayer radius and bubble volume are shown in Fig. 8 for four different conditions of heat flux and surface superheat at bubble inception $\Delta T_{\mathrm{i}}$. The experimental data from Nakamura and Utaka [4] were adopted for comparison. For all the results (minimum mesh size of $2.5 \mu \mathrm{m}$ ) given in Fig. 8, the microlayer radius increased sharply during the early stage of bubble growth, then decreased gradually after reaching maximum values. For three groups of results out of four (except for $q=76 \mathrm{~W} / \mathrm{m}^{2}$ and $\Delta T_{\mathrm{i}}=10 \mathrm{~K}$ ), the experimental and numerical results for the maximum value of the microlayer radius were approximately coincident. Although the variations in both the simulated and experimental microlayer radius showed a similar trend, delayed growth appeared in the numerical simulation. Corresponding delays in the bubble volume growth were also revealed. The delay of bubble growth may be caused by the small difference in the initial temperature distribution of the liquid region between the simulation and experiment, and the neglect of the drag force induced by the wake flow left by the previously detached bubble.

For the condition of $q=76 \mathrm{~W} / \mathrm{m}^{2}$ and $\Delta T_{\mathrm{i}}=10 \mathrm{~K}$, the calculation results for the microlayer radius and vapor volume indicate slower growth and a smaller maximum value than the experimental results. The 
possible reason for this is that $\Delta T_{\mathrm{i}}=10 \mathrm{~K}$ is different from the average value (as shown in Fig. 1); the temperature distribution applied in the simulation using the method introduced in chapter 2.5 may differ considerably from the actual temperature distribution. Consequently, except for the condition that differed significantly from the average state, it could be concluded that similar tendencies were observed for the experimental and calculation results, although some quantitative differences remained. However, it was confirmed that the growth and detachment process of boiling bubbles can generally be simulated using the calculation method proposed in this study.

Nakamura and Utaka [4] showed that the different growth curves for the microlayer radius became coincident after nondimensionalization. Similar characteristics of microlayer growth were evident in the present study. Figure 9 shows a comparison of the calculation results for the microlayer radius from Fig. 8 with the experimental results in a nondimensional form. Although some delay exists in the growth process from the calculation results, an approximately similar tendency is shown for the experimental results. In the simulations, the average temperature distribution in the liquid region was applied. However, the actual temperature distribution has temporal and spatial variation induced by mixing of the superheated and bulk saturated liquid with the movement of bubbles, especially in the superheated liquid layer in the vicinity of the heat transfer surface. Therefore, it is difficult to obtain the initial temperature distribution for the simulations as that experimentally measured. Thus, it is difficult to obtain calculation results that exactly coincide with the experimental results. Therefore, further effort is necessary to improve the initial temperature distribution in the numerical simulation.

The experimental images of boiling bubbles and the calculated bubble shapes during the bubble growth process shown in Fig. 10 ( $q=103 \mathrm{~W} / \mathrm{m}^{2}$ and $\Delta T_{\mathrm{i}}=24 \mathrm{~K}$ ) confirm that approximately similar bubble shapes can be achieved by numerical simulation in comparison with the experiments.

\subsection{Temperature distribution of liquid in the vicinity of the bubble interface}

The evaporation at the vapor-liquid interface is closely related to the temperature distribution of the liquid in the vicinity of the interface during the bubble growth process. Therefore, the liquid temperature distribution in the vicinity of the bubble interface was investigated. Isothermal diagrams for the vicinity of the bubble base are shown in Fig. 11 for example conditions of $q=103 \mathrm{~W} / \mathrm{m}^{2}$ and $\Delta T_{\mathrm{i}}=24 \mathrm{~K}$. It is confirmed that the liquid temperature approaches the saturation temperature when nearing the vapor-liquid interface and a temperature gradient is induced by the evaporation. The temperature gradient is larger when the position is closer to the heat transfer surface, because the liquid superheat is larger when closer to the heat transfer surface. A larger temperature gradient is thus induced by the more rapid evaporation. Moreover, the high temperature liquid near the vapor-liquid interface moves farther from the heat transfer surface along the vapor-liquid interface with bubble growth. As a result, a high temperature region is formed in the vicinity of the vapor-liquid interface near the bubble base, as shown in Fig. 11. 


\subsection{Heat transfer characteristics of microlayer evaporation}

The variations in the microlayer thickness distribution, the temperature of the heat transfer surface and the heat flux for microlayer evaporation under the condition of $q=103 \mathrm{~W} / \mathrm{m}^{2}$ and $\Delta T_{\mathrm{i}}=24 \mathrm{~K}$ are shown in Figs. 12-14, respectively. From Fig. 12, it is confirmed that the microlayer grows and becomes thinner with time due to evaporation, and dryout (thickness is zero) occurs initially at the center of the microlayer and then extends towards the outer region. Corresponding to the variation in microlayer thickness, the superheat of the heat transfer surface decreases rapidly with evaporation of the microlayer and then recovers after dryout occurs, as shown in Fig. 13. The heat flux distribution for microlayer evaporation is shown in Fig. 14. As the microlayer radius increases, there is larger heat flux for microlayer evaporation in the outer margin of the microlayer, because the high temperature heat transfer surface is continuously exposed with the increase in the microlayer radius; higher heat flux for evaporation is thus achieved in the newly exposed region of the microlayer. The higher heat flux region disappears after bubble detachment starts and the microlayer radius decreases, and then the exposed high temperature heat transfer surface is covered by bulk liquid again. Furthermore, for the entire microlayer region, a higher heat flux of evaporation was achieved during the early stage of bubble growth, and the heat flux of evaporation for the entire microlayer region then decreases with time. For each stage of bubble growth shown in Fig. 14, an extremely narrow region with a sharp increase in heat flux can be observed in the inner region of the microlayer (the connection of the microlayer and dryout regions). The reasons for the existence of the extremely small region are: 1) the rapid increase in heat flux with the decrease in microlayer thickness, especially for the extremely thin microlayer region, because the heat flux of the microlayer evaporation has an inverse relationship with the microlayer thickness, and 2) the heat supplied by heat conduction from the relatively high temperature dryout region. Figure 15 shows the relation between the heat flux for microlayer evaporation and the microlayer thickness for various superheat temperatures of the heat transfer surface, $\Delta T_{\text {sat }}$. For the same superheat of the heat transfer surface $\Delta T_{\text {sat }}$, the heat flux for microlayer evaporation increases with a decrease in the microlayer thickness. In particular, for a microlayer thickness $\delta$, less than $0.5 \mu \mathrm{m}$, the heat flux increases sharply with the decrease in microlayer thickness. For the narrow region of sharply increasing heat flux shown in Fig. 14, the superheat temperature of the heat transfer surface is below $0.1 \mathrm{~K}$ and the microlayer thickness is thinner than 0.1 $\mu \mathrm{m}$, which is in the high heat flux region, as shown in Fig. 15.

\subsection{Ratio of microlayer evaporation to total rate of evaporation of the bubble}

The variations in microlayer evaporation and total evaporation for the period from bubble inception to detachment are shown in Figs. 16(a) and (b) for two heat flux conditions of $q=76$ and $103 \mathrm{~kW} / \mathrm{m}^{2}$. Evaporation from the microlayer and the total amount of evaporation are presented as time integration values. Similar tendencies are observed for the variations in microlayer evaporation and the total evaporation under all calculation conditions. The ratio of microlayer evaporation to the total rate of 
evaporation of the bubble $\left(V_{\mathrm{ML}} / V_{\mathrm{B}}\right)$, which was calculated based on the time integration values of $V_{\mathrm{ML}}$ and $V_{\mathrm{B}}$, are also shown in Figs.16 (a) and (b). Although a small change is evident, it could be concluded that the ratio of microlayer evaporation remains almost constant until bubble detachment.

Figure 17 shows the ratio of microlayer evaporation $V_{\mathrm{ML}}$ to the total amount of evaporation $V_{\mathrm{B}}$ (bubble volume) for the period from bubble inception to detachment. Previous experimental results [7] are shown for comparison. For the calculation conditions close to the averaged value of $\Delta T_{\mathrm{i}}$, such as $\Delta T_{\mathrm{i}}=19 \mathrm{~K}$ for $q=76 \mathrm{~kW} / \mathrm{m}^{2}$, and $\Delta T_{\mathrm{i}}=24$ and $26 \mathrm{~K}$ for $q=103 \mathrm{~kW} / \mathrm{m}^{2}$, the ratios of $V_{\mathrm{ML}}$ to $V_{\mathrm{B}}$ were approximately 38, 44 and $44 \%$, respectively. The $V_{\mathrm{ML}} / V_{\mathrm{B}}$ ratios are approximately coincident with the previous results [7]. However, for the calculation conditions that deviate from the averaged value of $\Delta T_{\mathrm{i}}$, e.g. $\Delta T_{\mathrm{i}}=10 \mathrm{~K}$ for $q=76 \mathrm{~kW} / \mathrm{m}^{2}$, a higher ratio of microlayer evaporation was obtained for the calculation result in this study. As discussed in chapter 3.1, the possible reason for this is the difference between the calculation and experiment of initial temperature distributions in the liquid region. Consequently, based on the results near the averaged $\Delta T_{\mathrm{i}}$, the evaporation from the microlayer makes a large contribution to the bubble growth in nucleate pool boiling. The $V_{\mathrm{ML}} / V_{\mathrm{B}}$ ratio increases with higher surface superheat at bubble inception $\Delta T_{\mathrm{i}}$, which is in approximate agreement with the tendency observed in ref. [7].

\section{Conclusions}

Numerical simulations were conducted for the growth of a single bubble during nucleate pool boiling of water; a special calculation model based on the VOF method was adopted that considers the microlayer evaporation and experimentally measured microlayer thickness distribution. The following conclusions were made.

(1) The process from bubble inception to detachment could be simulated by numerical calculation.

(2) Similar tendencies were observed for the variations in microlayer radius and bubble volume for the simulation in this study and previous experimental results. The maximum value of microlayer radius was also approximately coincident with the experimental results.

(3) Bubble growth was affected by the temperature distribution in the superheated liquid layer. There is a higher temperature gradient at the vapor-liquid interface, which is closer to the heat transfer surface.

(4) The microlayer becomes thinner and dryout occurs initially at the center of the microlayer, and then extends to the outer region with the development of evaporation. The temperature of heat transfer surface decreases rapidly and then recovers after dryout occurs. In addition, the heat flux for microlayer evaporation increases with the microlayer radius and has a maximum in the periphery of the microlayer.

(5) Microlayer evaporation plays an important role during the bubble growth process during nucleate pool boiling. For the period from bubble inception to detachment, the ratio of microlayer evaporation $V_{\mathrm{ML}}$, to the total amount of evaporation $V_{\mathrm{B}}$, is approximately $40 \%$ for the calculation conditions employed in this study. 


\section{References}

[1] R. Sharp, The nature of liquid film evaporation during nucleate boiling, NASA TN D-1997, Lewis Research Center, Cleveland, Ohio (1964).

[2] L.D. Koffman, M.S. Plesset, Experimental observations of the microlayer in vapor bubble growth on a heated solid, J. Heat Transfer, 105 (1983), 625-632.

[3] Y. Utaka, K. Nakamura, A. Sakurai, K. Itagaki, A. Sonoda, Configuration of microlayer in nucleate boiling, Trans. Jpn. Soc. Mech. Eng. (Ser. B), 74(747) (2008) 2358-2364.

[4] K. Nakamura, Y. Utaka, Heat transfer characteristics based on micro-layer configuration in nucleate boiling, Trans. Jpn. Soc. Mech. Eng. (Ser. B), 74(748) (2008) 2560-2567.

[5] Y. Utaka, Y. Kashiwabara, M. Ozaki, Microlayer structure in nucleate boiling of water and ethanol at atmospheric pressure, Int. J. Heat Mass Transfer, 57 (2013) 222-230.

[6] T. Yabuki, O. Nakabeppu, On heat transfer mechanism of nucleate boiling with MEMS sensors (3rd Report, evaluation of the approach method and heat transfer characteristics of isolated boiling bubble), Trans. Jpn. Soc. Mech. Eng. (Ser. B), 76(771) (2010) 1932-1941.

[7] Y. Utaka, Y. Kashiwabara, M. Ozaki, Z. Chen, Heat transfer characteristics based on microlayer structure in nucleate pool boiling for Water and Ethanol, Int. J. Heat Mass Transfer, 68 (2014) 479-488.

[8] C.W. Hirt, B.D. Nichols, Volume of fluid (VOF) method for the dynamics of free boundaries, J. Comput. Phys., 39 (1981) 201-225.

[9] P. Stephan, J. Hammer, A new model for nucleate boiling heat transfer, Warme and Stoffubertragung, 30 (1994) 119-125.

[10] C. Kunkelmann, P. Stephan, CFD simulation of boiling flows using the Volume-Of-Fluid method within open foam, Numer. Heat Transfer, Part A, 56 (2009) 631-646.

[11] Y. Ose, Z. Kawara, T. Kunugi, Numerical simulation on subcooled boiling bubble behavior, Prog. Multiphase Flow Res., 4 (2009) 29-36.

[12] I. Ohnaka, Introduction to computational analysis of heat transfer and solidification -application to the casting processes-, 202, Maruzen, Tokyo (1985).

[13] Y.Y. Jiang, H. Osada, M. Inagaki, N. Horinouchi, Dynamic modeling on bubble growth, detachment and heat transfer for hybrid-scheme computations of nucleate boiling, Int. J. Heat Mass Transfer, 56 (2013) 640-652.

[14] A. Sanna, T.G. Karayiannis, D.B.R. Kenning, et al., Steps towards the development of an experimentally verified simulation of pool nucleate boiling on a silicon wafer with artificial sites, Appl. Therm. Eng., 29(7) (2009) 1327-1337.

[15] Fluent 6.3 User’s Guide, Fluent Inc. Lebanon, NH, 2006.

[16] D.L. Youngs. Time-dependent multi-material flow with large fluid distortion. In: K.W. Morton, M.J. Baines, editors, Numerical Methods for Fluid Dynamics, Academic Press, 1982, pp. 273-285. 
[17] J.U. Brackbill, D.B. Kothe, C. Zemach. A Continuum method for modeling surface tension. J. Comput. Phys., 100 (1992) 335-354.

[18] R.I. Issa, Solution of the implicitly discretized fluid flow equations by operator-splitting, J. Comput. Phys., 62 (1985) 40-65.

[19] J.B. Haelssig, A.Y. Tremblay, J. Thibault, S.G. Etemad, Direct numerical simulation of interphase heat and mass transfer in multicomponent vapour-liquid flows, Int. J. Heat Mass Transfer, 53 (2010) 3947-3960.

[20] Y. Fujita, K. Nishikawa, K. Hirahaya, Study on thermal boundary layer at heat transfer in nucleate pool boiling, Trans. Jpn. Soc. Mech. Eng., 43(376) (1977) 4586-4579. 
Figure Captions

Fig. 1. Contribution of evaporation from the microlayer as a function of surface superheat at bubble inception.

Fig. 2. Calculation domain.

Fig. 3. Schematic diagram of the vapor-liquid interface for a microlayer.

Fig. 4. Schematic diagrams of the vapor-liquid interface in bulk liquid.

Fig. 5. Schematic diagram of the apparent contact angle.

Fig. 6. Variation in apparent contact angle as a function of bubble time.

Fig. 7. Variation in microlayer radius as a function of bubble time for various minimum mesh sizes.

Fig. 8. Variation in microlayer radius and vapor volume as a function of bubble time.

Fig. 9. Variation in dimensionless microlayer radius as a function of dimensionless time.

Fig. 10. Comparison of experimental and calculated bubble shapes.

Fig. 11. Isothermal diagrams of liquid in the vicinity of the bubble base.

Fig. 12. Distributions of microlayer thickness as a function of radius for various bubble times.

Fig. 13. Distributions of surface superheat as a function of radius for various bubble times.

Fig. 14. Distributions of heat flux for microlayer evaporation as a function of radius for various bubble times.

Fig. 15. Heat flux of microlayer evaporation as a function of microlayer thickness for various surface superheat temperatures.

Fig. 16. Amount of evaporation from the microlayer and the total evaporation as a function of the bubble time.

Fig. 17. Comparison of the contribution from microlayer evaporation with previous results. 


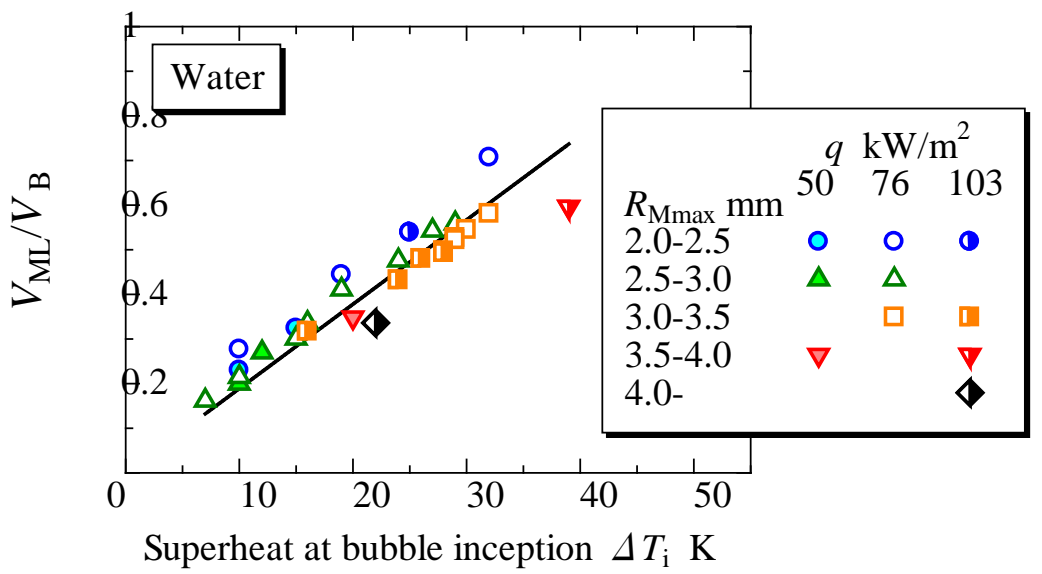

Fig. 1. Contribution of evaporation from the microlayer as a function of surface superheat at bubble inception. 


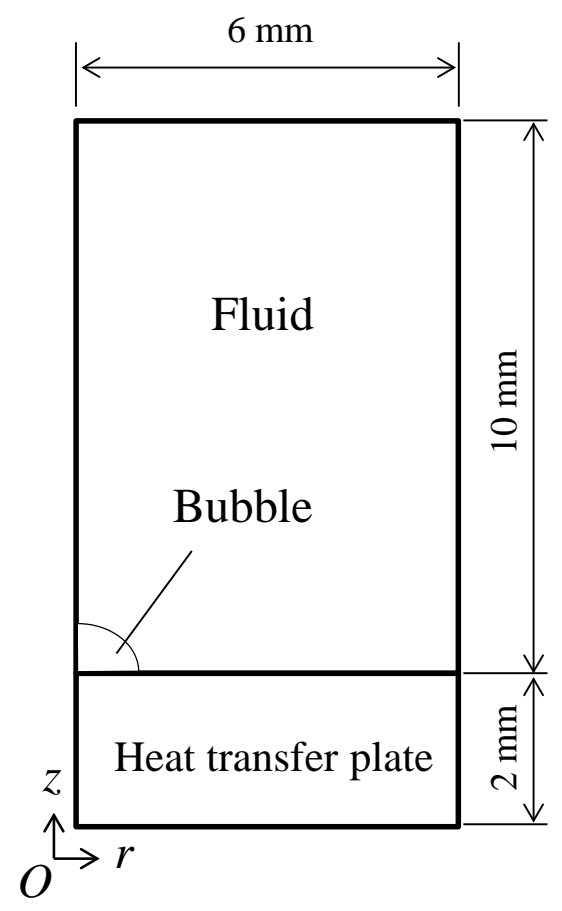

Fig. 2. Calculation domain. 


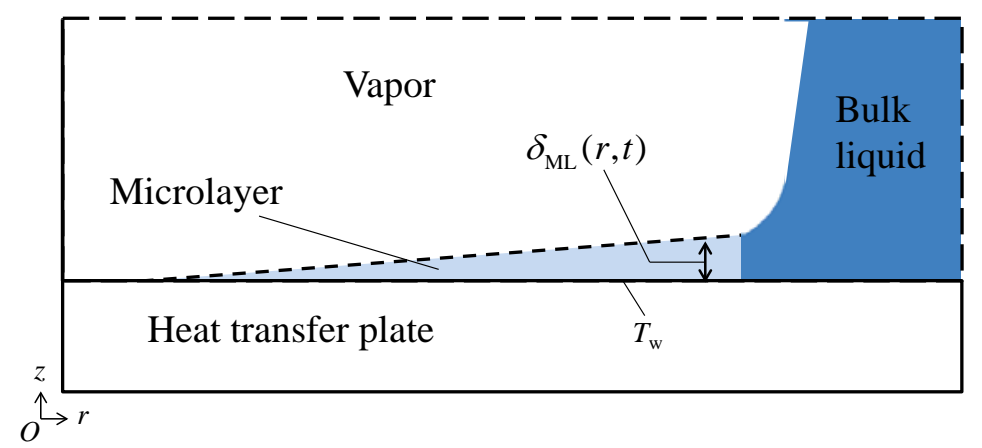

Fig. 3. Schematic diagram of the vapor-liquid interface for a microlayer. 


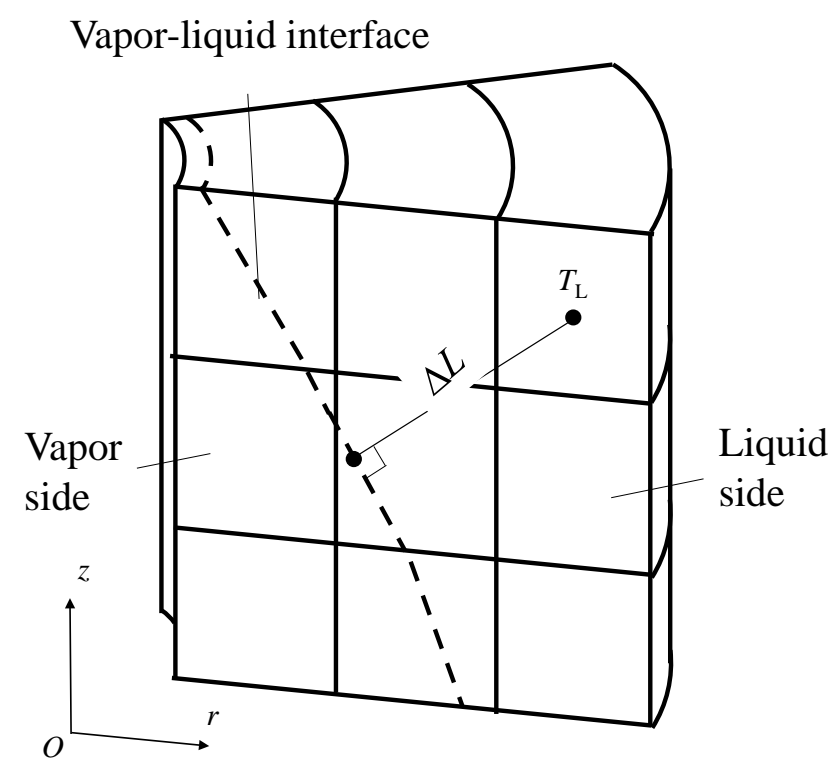

(a)

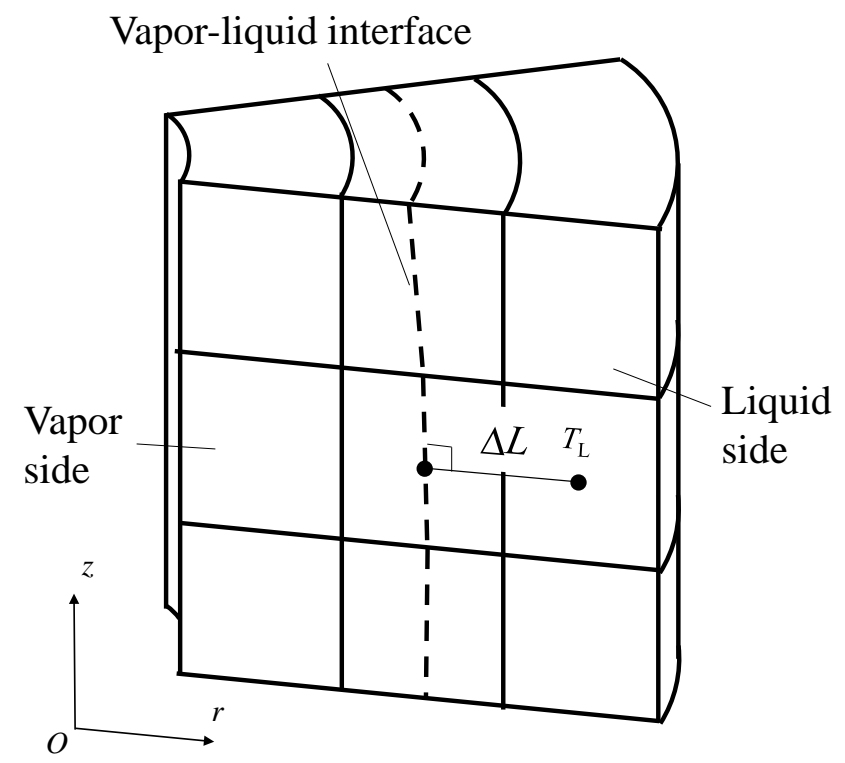

(b)

Fig. 4. Schematic diagrams of the vapor-liquid interface in bulk liquid. 


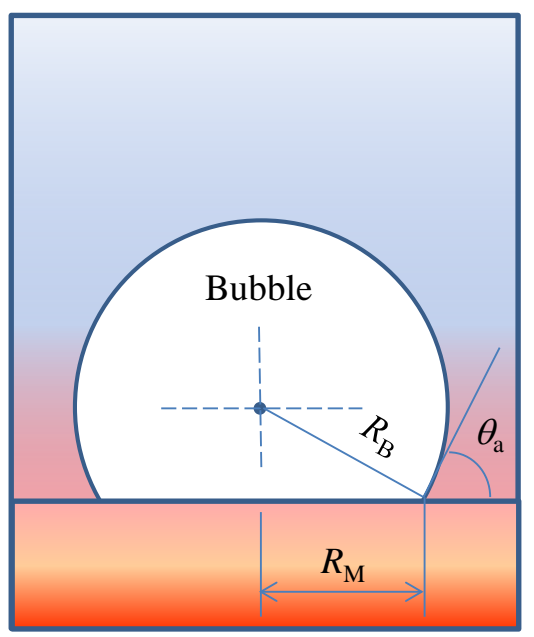

Fig. 5. Schematic diagram of the apparent contact angle. 


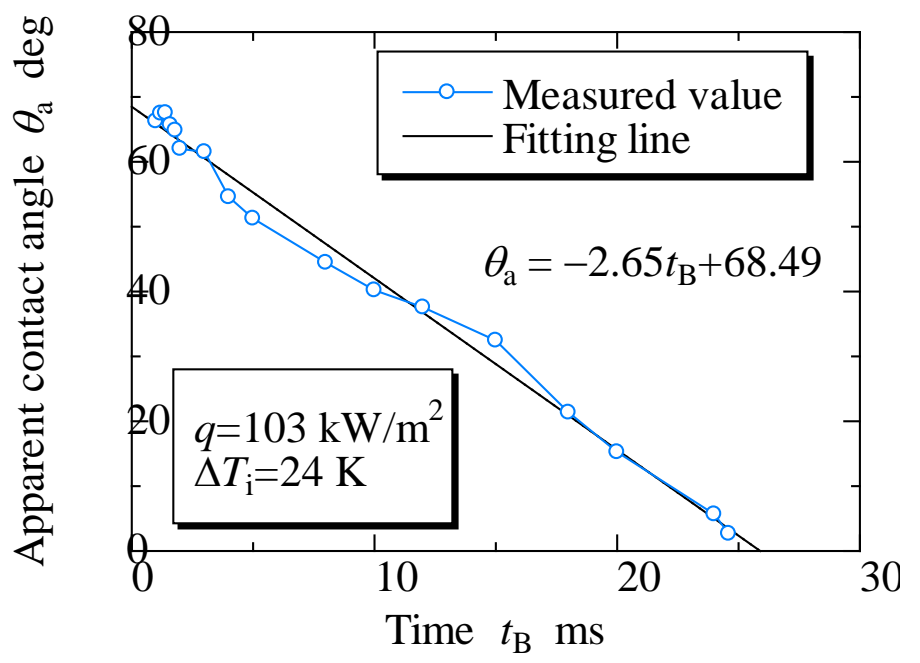

Fig. 6. Variation in apparent contact angle as a function of bubble time. 


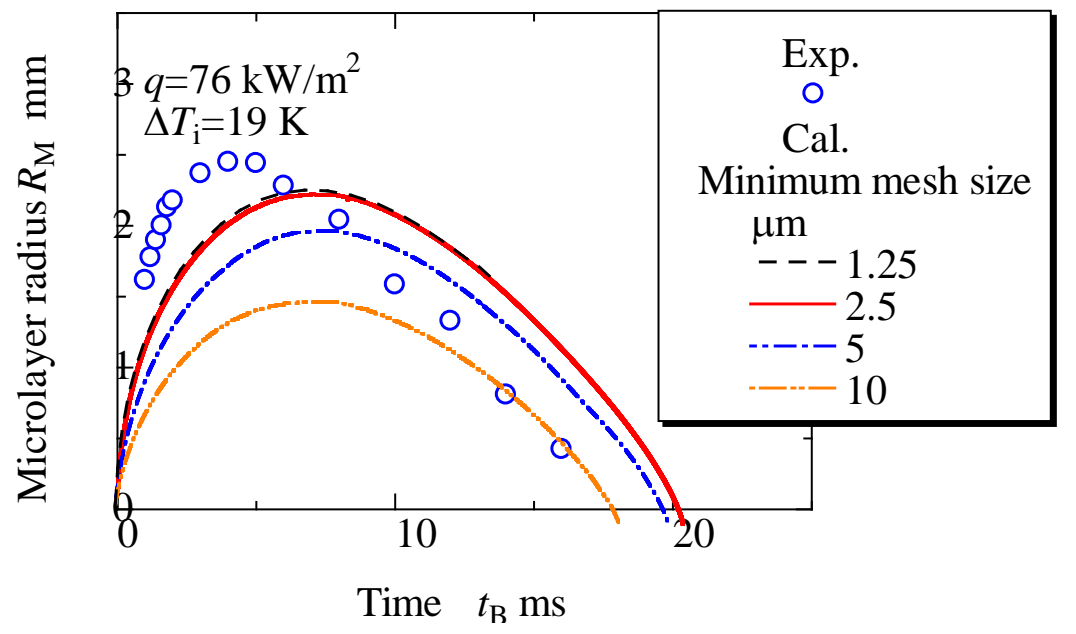

Fig. 7. Variation in microlayer radius as a function of bubble time for various minimum mesh sizes. 


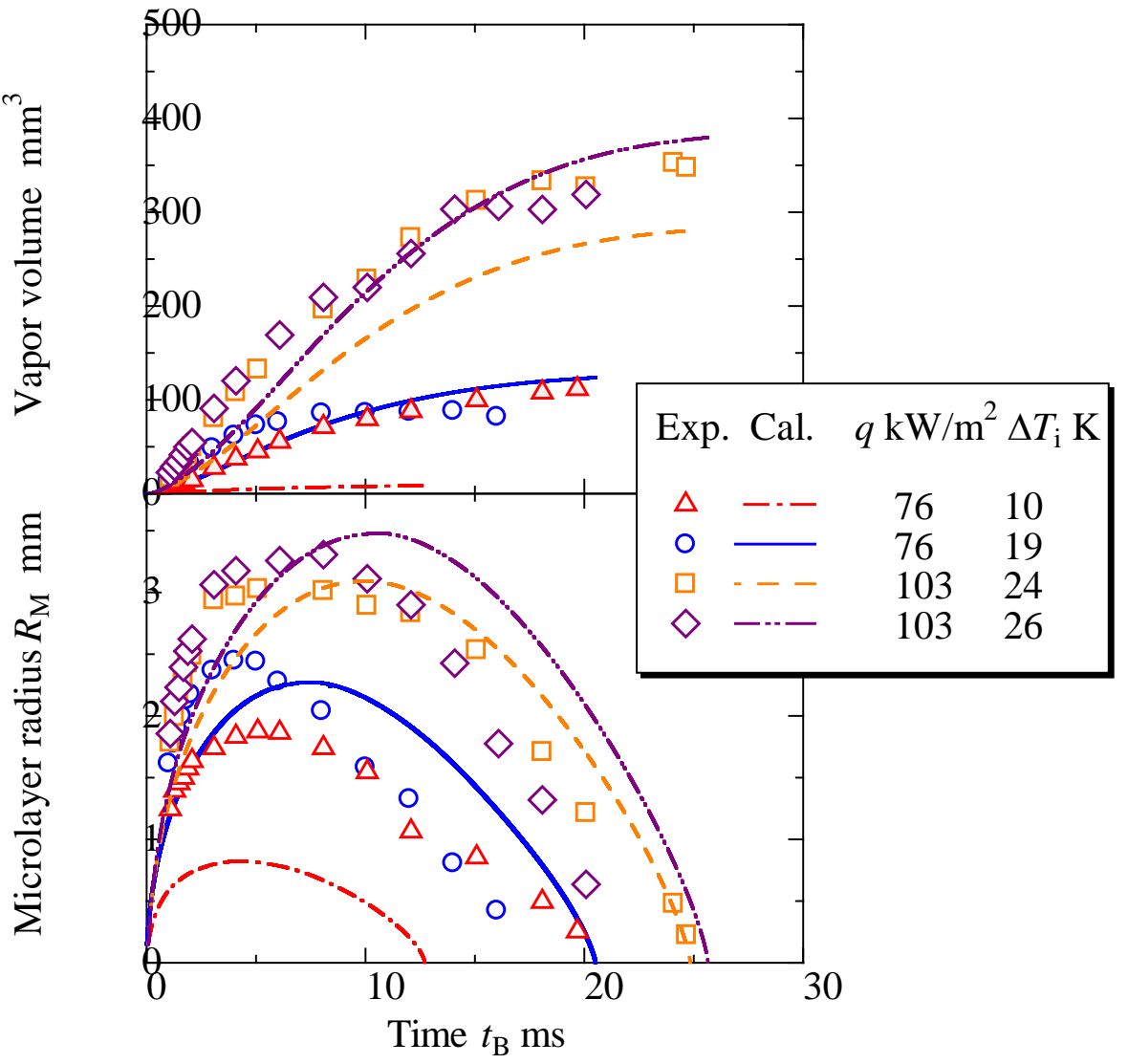

Fig. 8. Variation in microlayer radius and vapor volume as a function of bubble time. 


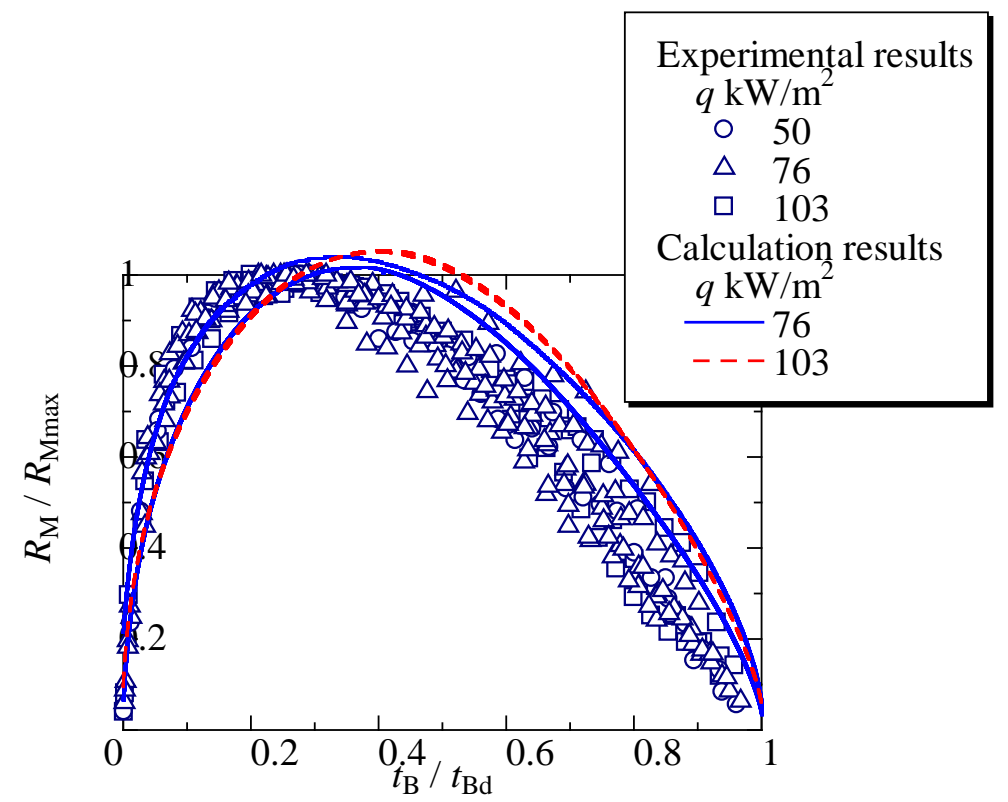

Fig. 9. Variation in dimensionless microlayer radius as a function of dimensionless time. 


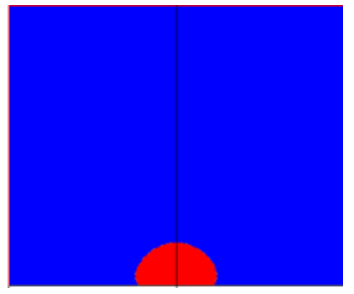

$2 \mathrm{~mm}$ $t_{\mathrm{B}}=1.0 \mathrm{~ms}$

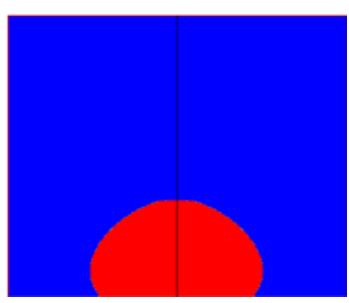

$2 \stackrel{\mathrm{mm}}{\longleftrightarrow}$

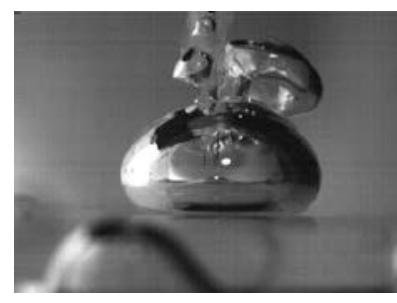

$2 \underset{\longleftrightarrow m}{\longleftrightarrow}$

$t_{\mathrm{B}}=5.1 \mathrm{~ms}$

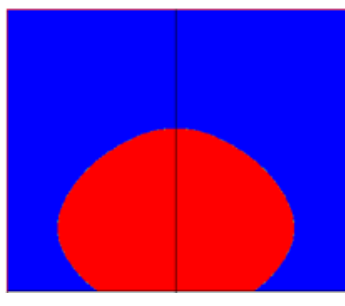

$2 \stackrel{\mathrm{mm}}{\longleftrightarrow}$

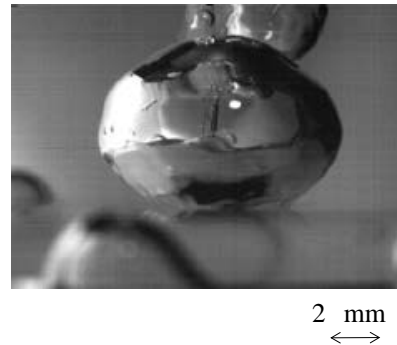

$t_{\mathrm{B}}=14.8 \mathrm{~ms}$

Fig. 10. Comparison of experimental and calculated bubble shapes. 


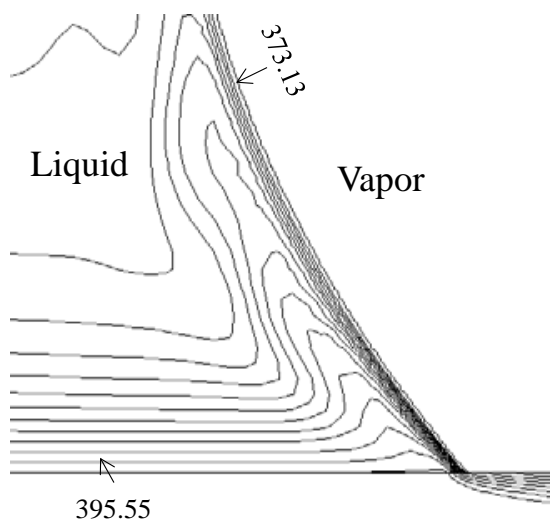

(a) $t_{\mathrm{B}}=5.1 \mathrm{~ms}$

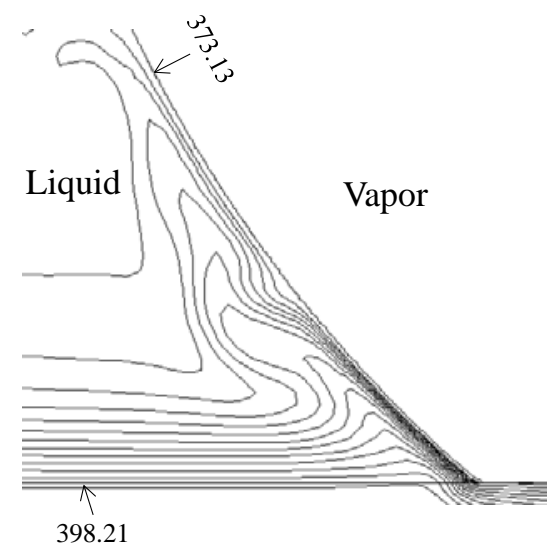

(b) $t_{\mathrm{B}}=10.2 \mathrm{~ms}$

Fig. 11. Isothermal diagrams of liquid in the vicinity of the bubble base. 


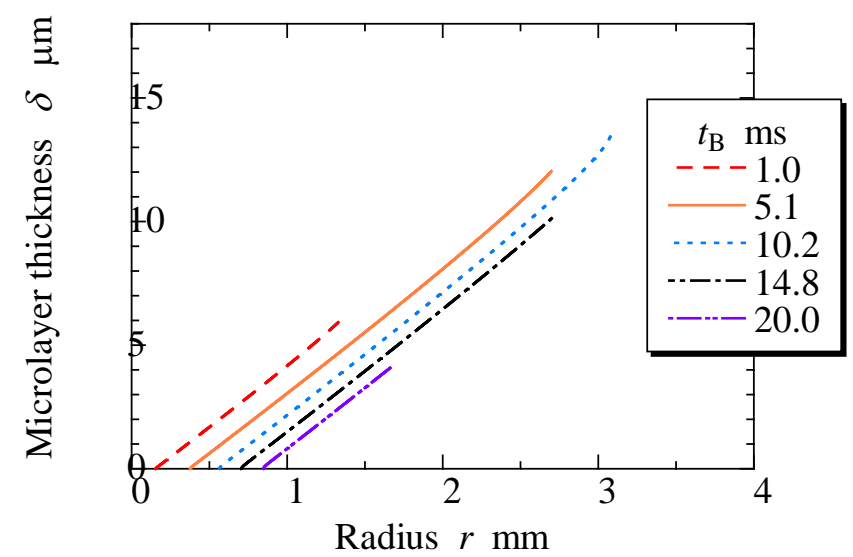

Fig. 12. Distributions of microlayer thickness as a function of radius for various bubble times. 


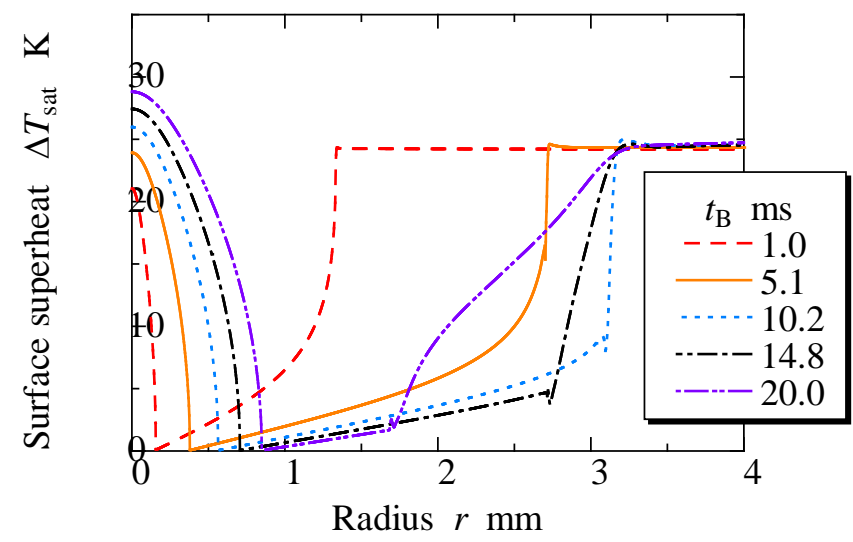

Fig. 13. Distributions of surface superheat as a function of radius for various bubble times. 


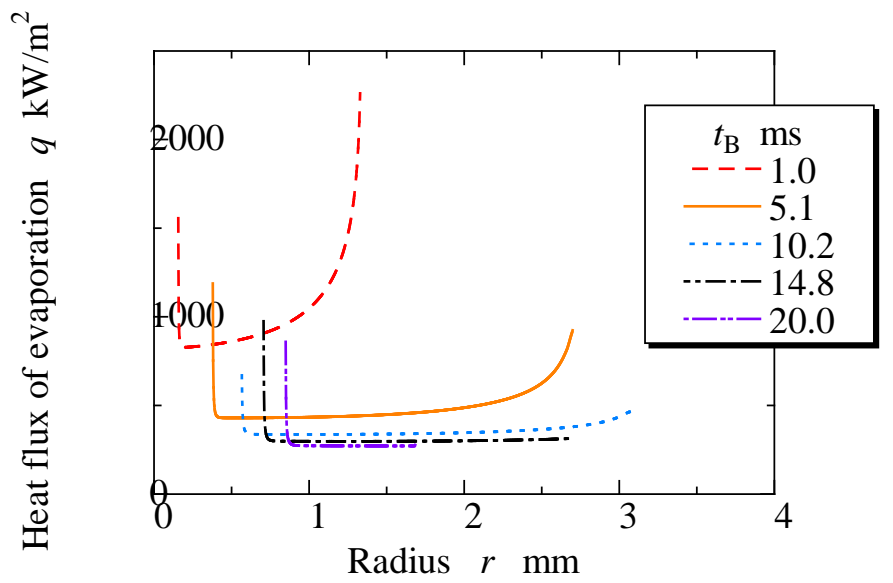

Fig. 14. Distributions of heat flux for microlayer evaporation as a function of radius for various bubble times. 


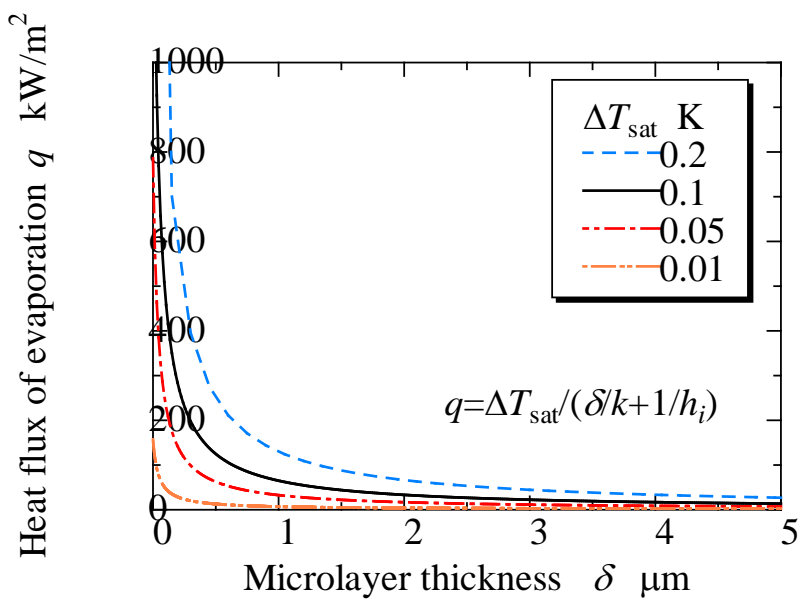

Fig. 15. Heat flux of microlayer evaporation as a function of microlayer thickness for various surface superheat temperatures. 


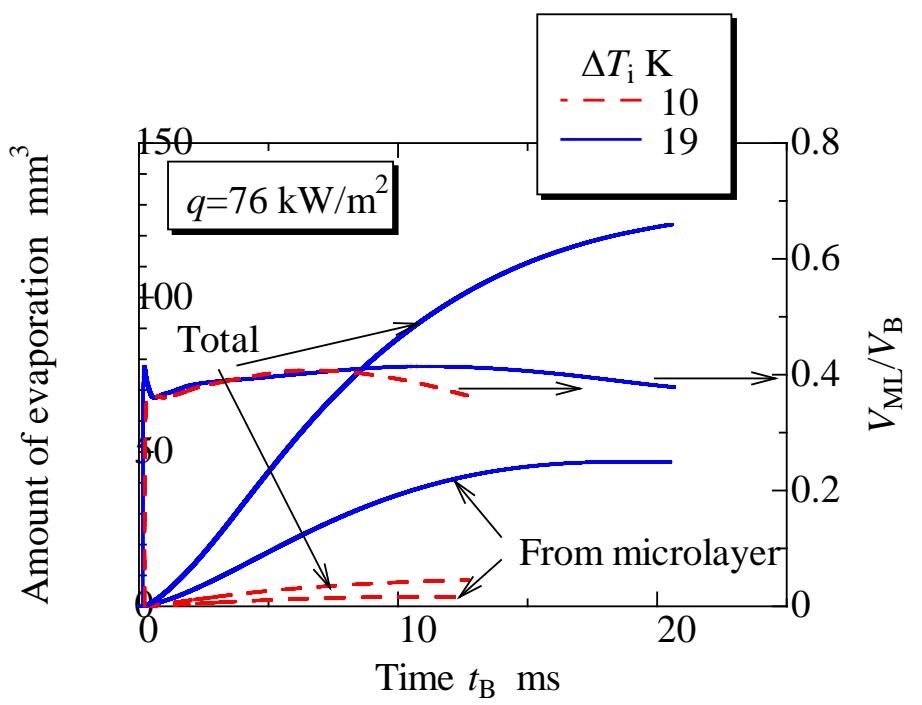

(a) $q=76 \mathrm{~kW} / \mathrm{m}^{2}$

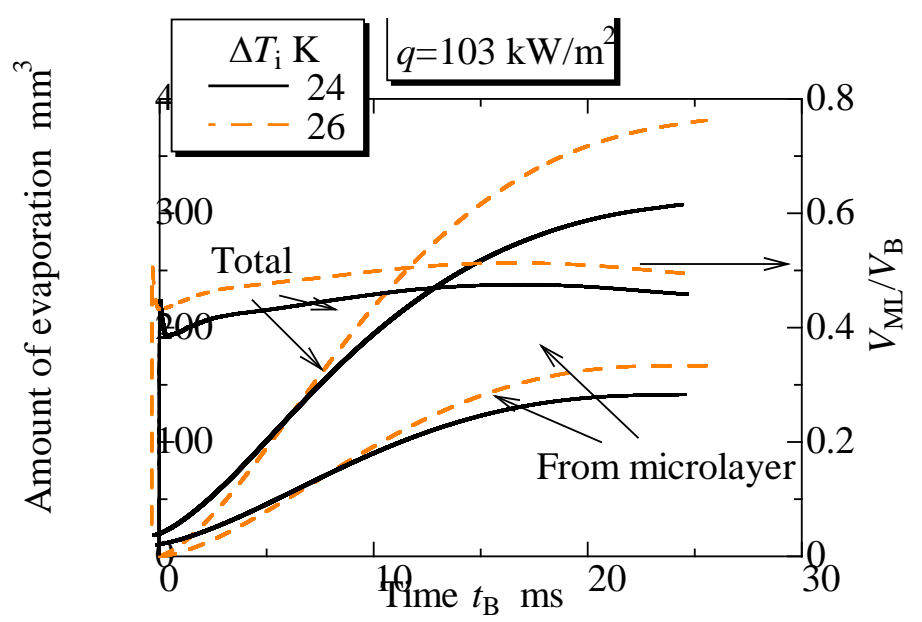

(b) $q=103 \mathrm{~kW} / \mathrm{m}^{2}$

Fig. 16. Amount of evaporation from the microlayer and the total evaporation as a function of the bubble time. 


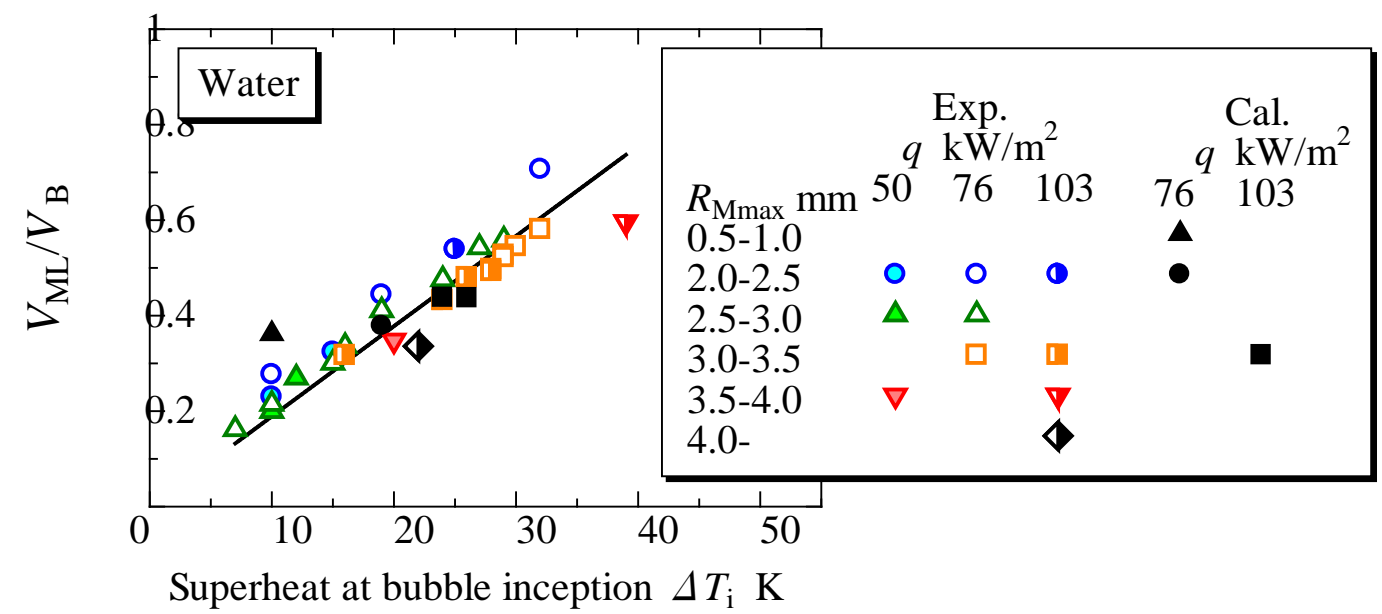

Fig. 17. Comparison of the contribution from microlayer evaporation with previous results. 